\title{
Guest Editorial: Smart Grid Inspired Data Sensing, Processing and Networking Technologies
}

\author{
Jia $\mathrm{Hu}^{1} \cdot$ Kun Yang ${ }^{2,3} \cdot$ Victor C. M. Leung ${ }^{4} \cdot \mathrm{Ke} \mathrm{Xu}^{5}$ \\ Published online: 14 September 2019 \\ (C) Springer Science+Business Media, LLC, part of Springer Nature 2019
}

\section{Editorial:}

The smart grid is the next generation electric grid that enables efficient, intelligent, and economical power generation, transmission, and distribution using modern information and communications technologies, such as advanced sensing, monitoring, and processing technology, and high-speed bi-directional communications and networking. In recent years, the smart grid has attracted significant attentions and become a global trend due to the immense potential benefits including enhanced reliability and resilience, higher operational efficiency, more efficient energy consumption, and better power quality. A long-term strategy to address various challenges introduced or inspired by smart grid shall be a systematic one that brings together the three key aspects of a smart grid communications system that is the core of smart grid, namely, data sensing, data processing and data communications. It is precisely the purpose of this special issue, which serves an integration of these major components or enabling technologies of smart grid.

This special issue features five selected high-quality papers. The first paper entitled "Mobility-aware Vehicle-toGrid (V2G) Optimization for Uniform Utilization in Smart Grid based Power Distribution Network" proposed a scalable virtual power plant (VPP) based V2G optimization architecture integrated with VANET. Simulations showed that the proposed mobility-aware and scalable V2G optimization

Jia $\mathrm{Hu}$

J.Hu@exeter.ac.uk

1 Department of Mathematics and Computer Science, Liverpool Hope University, Liverpool, UK

2 Department of Electronic \& Electrical Engineering, University College London (UCL), London, UK

3 School of Computer Science \& Electronic Engineering, University of Essex, Essex, UK

4 University of British Columbia (UBC), Vancouver, Canada

5 Department of Computer Science, Tsinghua University, Beijing, China

algorithm can reduce or significantly postpone the need of expensive upgrade of power distribution infrastructure.

In the second paper with the title "Incorporating FAIR into Bayesian Network for Numerical Assessment of Loss Event Frequencies of Smart Grid Cyber Threats", the authors proposed a method to incorporate the FAIR's LEF into Bayesian Network (BN) to derive the numerical assessments to rank the threat severity. The improvements were demonstrated by applying the method to assess cyber threats in a smart grid robustness research project (IRENE).

In the paper entitled "A Decision Function Based Smart Charging and Discharging Strategy for Electric Vehicle in Smart Grid", the authors proposed an Electric Vehicle Charging and Discharging Strategy (EVCDS) based on a Charging Decision Function (CDF) as well as a Discharging Decision Function (DDF). Comparing with other strategies, EVCDS performs well in terms of reducing the charging demand fluctuations and improving the charging demand balance among charging stations.

The next paper entitled "Efficient Identification of TOP-K Heavy Hitters over Sliding Windows" proposed a new data structure called FCM and its associated algorithms. The key point of this method is that it introduces a novel filter-and-jump mechanism. The experimental results demonstrate that the performance of FCM is superior to the previous related algorithm.

The last paper entitled "LTE Delay Assessment for RealTime Management of Future Smart Grids" investigated the feasibility of using Long Term Evolution (LTE) cellular networks for the real-time smart grid state estimation. The results show that time-delay prioritized scheduling in combination with flexible PRB assignment greatly reduces the maximum delay when compared to simple random scheduling and fixed PRB assignment.

Acknowledgements The guest editors are grateful to the reviewers for their efforts in reviewing the manuscripts. We also thank the Editor-in-Chief, Dr. Imrich Chlamtac, for his support and guidance during the entire process.

Publisher's Note Springer Nature remains neutral with regard to jurisdictional claims in published maps and institutional affiliations. 


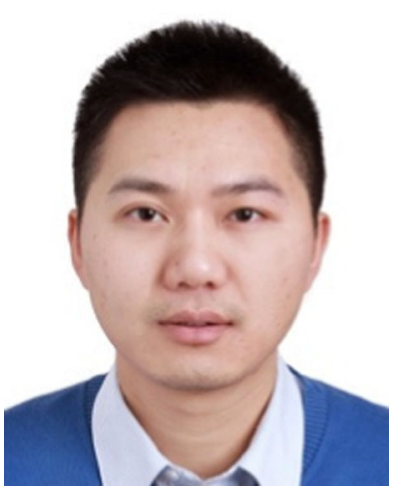

Dr. Jia $\mathrm{Hu}$ is a Lecturer at the Department of Computer Science, University of Exeter, $\mathrm{UK}$. He received his $\mathrm{PhD}$ in Computing from the University of Bradford, UK, in 2010. His research interests include performance modelling and analysis, next generation networking, resource allocation, and network security. He has published three book chapters and over 40 research papers within these areas in prestigious international journals (e.g., IEEE Trans. Wireless Communications, Information Sciences, Computer Networks) and at reputable international conferences (e.g., ICC, GLOBECOM, WCNC). He serves as the associate editor for Elsevier Computers \& Electrical Engineering and Hindawi. He also served as the guest editor for six major international journals. He has served as General Co-Chair of SONET'14, IEEE IUCC-AIPNGN 12-13, HPCC-AHPCN'12, FCSTAIPNGN'11, ICESS-ESA'10, Program Co-Chair of IEEE HPCC'12, Local Arrangement Chair of IEEE ICESS'10, and Publicity Chair of IEEE PICom'14. He received the Outstanding Service Award for contributions to IEEE ICESS ' 10 and HPCC'12.

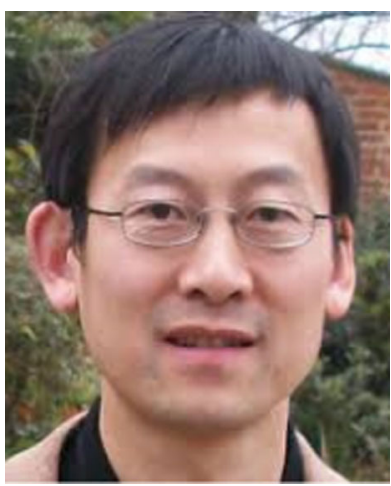

Professor Kun Yang received his PhD from the Department of Electronic \& Electrical Engineering of University College London (UCL), United Kingdom. He is currently a full Professor in the School of Computer Science \& Electronic Engineering, University of Essex, UK, heading the Network Convergence Laboratory (NCL). Before joining in University of Essex at 2003, he worked at UCL on several European Union (EU) research projects (such as FAIN, CONTEXT) for several years in the areas of IP network management, active networks and context-aware services. His main research interests include heterogeneous wireless networks, fixed mobile convergence, future Internet technology and network virtualization, cloud computing. He manages research projects funded by various sources such as UK EPSRC, EU FP7 and industries. He has published 70+ journal papers in addition to $60+$ conference papers. He is a Senior Member of IEEE and a Fellow of IET. He serves on the editorial boards of both IEEE and nonIEEE journals (Wiley and Springer) and have guest-edited several special issues in the above research areas including one in IEEE JSAC. He has also served as (co-)chair (general or TPC) in many IEEE conferences.

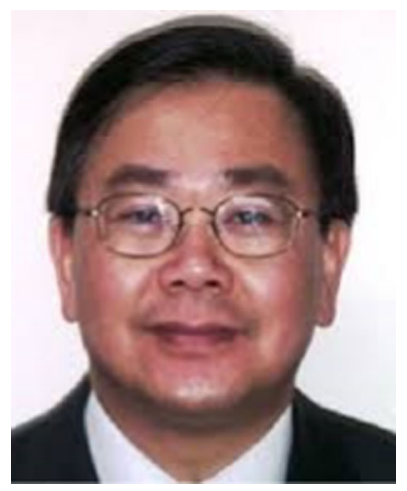

Professor Victor C. M. Leung is a Professor of Electrical and Computer Engineering and holder of the TELUS Mobility Research Chair at the University of British Columbia (UBC). His research is in the areas of wireless networks and mobile systems, where he has co-authored more than 700 technical papers in archival journals and refereed conference proceedings, several of which had won best-paper awards. Dr. Leung is a Fellow of IEEE, the Royal Society of Canada, the Canadian Academy of Engineering and the Engineering Institute of Canada. He is serving/has served on the editorial boards of IEEE JSAC, Transactions on Computers, Wireless Communications, and Vehicular Technology, Wireless Communications Letters, and several other journals. He has provided leadership to the technical committees and organizing committees of numerous international conferences. Dr. Leung was the recipient of an APEBC Gold Medal, NSERC Postgraduate Scholarships, a 2012 UBC Killam Research Prize, and an IEEE Vancouver Section Centennial Award.

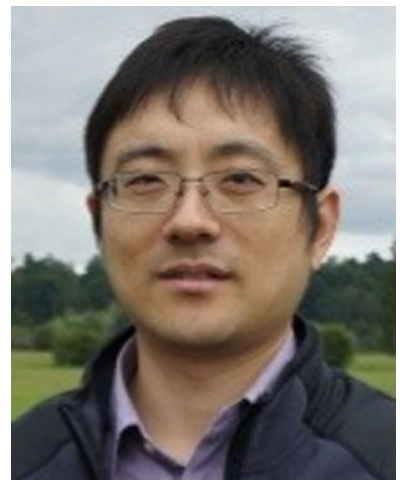

Professor Ke $\mathrm{Xu}$ received his Ph.D. from the Department of Computer Science of Tsinghua University. He is currently a full professor in the Department of Computer Science of Tsinghua University. He has published more than 100 technical papers and holds 20 patents in the research areas of next generation Internet, high performance router, Internet of Things (IoT), network virtualization and optimization. He has been a principal investigator of a few projects funded by the Chinese Ministry of Science and Technology and NSFC (National Science Foundation of China). Specifically, he studies the optimizationbased next generation Internet architecture such as optimal traffic engineering, optimal green routing. He is a Senior Member of IEEE and a member of ACM. He has guest edited several special issues in IEEE and Springer Journals. 